\title{
Performance of the colorectal cancer screening marker Sept9 is influenced by age, diabetes and arthritis: a nested case-control study
}

Mai-Britt W. Ørntoft ${ }^{1}$, Hans J. Nielsen ${ }^{2}$, Torben F. Ørntoft ${ }^{1}$, Claus L. Andersen ${ }^{1 *}$ and On behalf of the Danish Study Group on Early Detection of Colorectal Cancer

\begin{abstract}
Background: Annually, colorectal cancer (CRC) is diagnosed in $>1.4$ million subjects worldwide and incidence is increasing. Much effort has therefore been focused on screening, which has proven to reduce cancer-related mortality. The Sept9 DNA-methylation assay is among the most well studied blood-based screening markers. However, earlier reported performances may be misleading: the Sept9 test was recently examined in two screening based cohorts and yielded performances lower than expected. We hypothesize that comorbidities and/or demographic characteristics affect the results of the Sept9 test.

Methods: Using a retrospective nested case-control study design, we studied plasma from 150 cancer and 150 controls selected from a well-characterized cohort of 4698 subjects referred for diagnostic colonoscopy due to CRCrelated symptoms. The cases and controls were matched on age and gender, and moreover cases were stratified on tumor-site and tumor-stage. The selected cohort included a wide range of comorbidities. Plasma Sept9 levels were assessed using a commercially available PCR based assay (Epi-proColon).

Results: Clinical sensitivity for CRC stages I-IV was $37 \%, 91 \%, 77 \%$, and $89 \%$, and the overall sensitivity $73 \%(95 \% \mathrm{Cl}$, 64-80 \%) and specificity $82 \%(95 \% \mathrm{Cl}, 75-88 \%)$, respectively. Age $>65$ was associated with both increased false positive and false negative results $(p<0.05)$. Arthritis was associated with a higher false negative rate $(p=0.005)$ whereas Arteriosclerosis was associated with a higher false positive rate $(p=0.007)$. Diabetes was associated with Sept9 positivity with an OR of 5.2 (95\% Cl 1.4-19.1). When the performance of Sept9 was adjusted for these parameters in a final multivariate regression model, the OR for a positive Sept9 test to be associated with CRC increased from 8.25 (95\% Cl 4.83-14.09) to 29.46 (95 \% Cl 12.58-69.02).

Conclusions: The results indicate that the performance of the Sept 9 assay is negatively affected by several factors commonly associated with CRC screening populations: early-stage disease, age > 65 years, diabetes, arthritis, and arteriosclerosis. This should be taken into account if the Sept9 assay is used as a single marker for CRC screening, but may also have a wider impact, as it is likely that such factors may affect other blood based DNA markers as well.
\end{abstract}

Keywords: 3-10 words: Sept9, Colorectal cancer, Screening, DNA methylation marker, Comorbidities, Epigenetics

\footnotetext{
*Correspondence: cla@clin.au.dk

'Department of Molecular Medicine, MOMA, Aarhus University Hospital,

Skejby DK-8200Aarhus N, Denmark

Full list of author information is available at the end of the article
}

\section{Biomed Central}

(c) 2015 Ørntoft et al. Open Access This article is distributed under the terms of the Creative Commons Attribution 4.0 International License (http://creativecommons.org/licenses/by/4.0/), which permits unrestricted use, distribution, and reproduction in any medium, provided you give appropriate credit to the original author(s) and the source, provide a link to the Creative Commons license, and indicate if changes were made. The Creative Commons Public Domain Dedication waiver (http://creativecommons.org/publicdomain/zero/1.0/) applies to the data made available in this article, unless otherwise stated. 


\section{Background}

At present, primary colorectal cancer (CRC) is diagnosed in $>1.4$ million subjects annually and incidence is increasing [1]. Although improved surgical approaches for especially rectal cancer has improved the overall survival rates it is mainly early stage cancers that are curable by surgical intervention. The most reliable self-reported symptom of CRC cancer is dark rectal bleeding with a positive predictive value of 20.6 $\%$ and an odds ratio (OR) of 7.4 for CRC [2], but symptoms may be evasive. Therefore, much effort has been focused on screening and hence earlier detection of $\mathrm{CRC}$, which has repeatedly proven to reduce the cancer-related mortality $[3,4]$.

In recent years several screening markers have emerged to help diagnosing early stage CRC or even premalignant lesions. They separate in two different categories: stool markers, such as FOBT/FIT and bloodbased markers as DNA/RNA and proteins [5]. It is expected that blood-based screening assays may improve the clinical sensitivity compared with stool tests, because of an expected higher compliance among screening subjects [6,7]; however the cost-effectiveness has not yet been proven [8]. The Sept9 DNA-methylation test is among the most well studied blood tests; often in casecontrol study designs [9-11]. Recently, the Sept9 test was examined in two medium/large sized asymptomatic screening based cohorts and yielded overall sensitivities (range 48-68 \%) and specificities (range 78-91 \%) lower than expected based on the earlier case-control studies $[12,13]$. This may indicate that the performance of the Sept 9 test is affected by covariates, such as comorbidities and/or demographic characteristics of the subjects in screening populations. However, currently we have only limited knowledge of which comorbidities or characteristic could be involved as this has not been part of earlier study objectives.

To address this question we have tested the Sept9 assay on a nested case-control cohort selected from a large well-characterized cohort of subjects referred to colonoscopy due to CRC symptoms; the subjects also had a wide range of co-morbidities.

\section{Methods}

\section{Human plasma samples}

The retrospective nested case-control cohort in the present study was selected from the prospective Danish Endoscopy II study, a multicenter trial, which from April 2010 to November 2012 recruited 4698 subjects referred to colonoscopy due to CRC related symptoms. All patients gave informed consent to participate and the study was approved by the The Danish National Ethics Committee (H-3-2009-110) and the Danish Data Protection Agency (2007-58-0015). Exclusion criteria were previous colonoscopy, previous CRC or adenoma, diagnosis with HNPCC (Hereditary Nonpolyposis Colorectal Cancer or Lynch Syndrome) or FAP (Familial adenomatous polyposis), previous or present extracolonic malignant disease, or age under 18. Just prior to colonoscopy all subjects had a blood sample collected. All clinical information was collected, including surgical/oncological intervention, as well as cancer TNM stage, and adenoma histology. Intervention followed the Danish Colorectal Cancer Group (DCCG) guidelines. Chronic diseases were divided into large disease groups: Hypertension, Diabetes (type I and II), manifest Arteriosclerosis (pooling former AMI, stroke due to thrombosis, chronic ischemic diseases in peripheral arteries and chronic ischemic heart diseases), respiratory diseases and Arthritis (active arthritis in more than one joint).

The nested case-control cohort consisted of 300 participants: 150 cases and 150 controls with no evidence of CRC disease (NED). Cases consisted of 21 high risk adenomas (size $\geq 1 \mathrm{~cm}$ and/or villous histology $>25 \%$ and/ or sessile-serrated polyps and/or high-grade neoplasia and/or $\geq 3$ adenomas), 35 stage I CRC, 35 stage II CRC, 30 stage III CRC and 29 stage IV CRC based on UICC criteria. To minimize confounding, cases and controls were matched by age and gender. Cases were selected with gender evenly distributed according to disease stage and with $1 / 3$ of tumors localized in the rectum, 1/3 in the proximal colon (coecum, ascending colon and right flexure), and 1/3 in the distal colon. Chronic disease and comorbidities were not included as exclusion criteria. For all participants, clinical follow up for further 3 years were obtained; as all Danish patients are registered with a personal computerized ID-number, and all hospital treatment is recorded in a national database, there were no participants lost to follow-up. A survey of the cohort identified the expected associations between comorbidities and life style factors, indicating the cohort is representative of future CRC screening cohorts (Additional file 1: Table S1).

For all 300 participants, plasma was isolated from ethylenediaminetetraacetic acid (EDTA) stabilized whole blood by double centrifugation at $10 \mathrm{~min}$ for $3000 \mathrm{~g}$ at room temperature. Plasma was then stored at $-80{ }^{\circ} \mathrm{C}$ under 24/7 electronic surveillance until isolation of circulating cell-free DNA (cfDNA).

\section{Sept9 test}

cfDNA isolation and analysis for presence of methylated Sept9 DNA was done using the Epi-ProColon kit as described by Potter et al. [13]: The Epi proColon test comprises the Epi proColon Plasma Quick kit, the Sensitive PCR kit, and Control kit. All analyses were done blinded to subject outcome and were performed by Epigenomics $\mathrm{GmbH}$, Berlin. Samples were processed in batches with 
a random distribution of cases and controls to avoid analytical bias, and negative and positive processing controls ensured validity of the test result. A minimum of 2 $\mathrm{ml}$ of plasma was provided for cfDNA isolation; one participant did not meet this requirement and was excluded. cfDNA was isolated from $3.5 \mathrm{ml}$ plasma for 139 participants, and from between $2.0-3.5 \mathrm{~mL}$ plasma from 160 participants; a total of 299 subjects (149 cases and 150 controls).

To isolate cfDNA we used the Plasma Quick kit, where plasma was mixed with $3.5 \mathrm{ml}$ of lysis buffer and incubated for $10 \mathrm{~min}$, after which magnetic beads and absolute ethanol were added; the sample was incubated on a rotator for $45 \mathrm{~min}$. Impurities were removed from the magnetic beads in a wash step. The purified DNA was then released from the beads in elution buffer and treated at $80{ }^{\circ} \mathrm{C}$ with a solution containing ammonium bisulfite for deamination of cytosine. The converted cfDNA was captured by use of magnetic beads, passed through a series of wash steps, and eluted in $60 \mu \mathrm{L}$ buffer. Samples were then analyzed for presence of methylated Sept9 DNA with the Sensitive PCR kit on a 7500 Fast Dx Real Time PCR device (Life Technologies). The assay was designed as a duplex real-time PCR for the methylated Sept $9 \gamma$ promoter and ACTB (actin, beta) as an internal reference to assess the integrity of each sample. PCR was performed in triplicate with $15 \mu \mathrm{L}$ template DNA per well and run for 45 cycles. We recorded PCR results from the 7500 Fast Dx software for ACTB and methylated Sept 9 for each of the triplicate reactions. The validity of each sample batch was determined on the basis of methylated Sept9 and ACTB cycle threshold $(\mathrm{Ct})$ values for the positive and negative controls. Samples were only deemed valid if the ACTB control was positive in all three replicates (had amplification curves detected within 45 cycles). Sept9 test results for individual samples were scored positive, if a Ct value was detected within 45 cycles. Samples were scored negative when no methylated Sept $9 \mathrm{Ct}$ value was reported for any of the 3 valid PCR replicates.

\section{Statistical methods}

We assessed the association in between all variables and also pair-wise between Sept 9 and all other available variables using Fishers exact tests. All tests were two-sided, and $p<0.05$ was considered statistically significant. Test for trend of increasing true positive results with increasing tumor stage was done using the Wilcoxon rank sum test. Univariate logistic regression models were used to assess the diagnostic power of all available variables for CRC. Bivariate models were used to assess the association between Sept9 and all other available variables, including if any of the available variables modified the effect of the Sept9 test. Finally a multivariate model was built to assess the adjusted diagnostic power of the Sept 9 assay in the context of all the variables affecting or associated with it. All reported models passed the HosmerLemeshow's goodness of fit test. All assumptions for the different analyses were fulfilled. STATA V.12.1 (StataCorp LP, Texas, USA) were used for all statistical analyses.

\section{Results \\ Clinical performance}

An overview of the demographic and co-morbidity characteristics of the included subjects is provided in Table 1. In this study the overall sensitivity of the Sept 9 test for detecting CRC was $73 \%$ (95\% CI, 64-80\%) vs $59 \%$ (95 \% CI 50-67 \%), using the $1 / 3$ and $2 / 3$ scoring algorithms, respectively. Clinical sensitivity for the individual CRC stages I-IV, using the $1 / 3$ and $2 / 3$ algorithms was as follows: $37 \%$ vs $17 \%$; $91 \%$ vs $74 \%$; $77 \%$ vs $63 \%$; and $89 \%$ vs $86 \%$. The sensitivity was significantly lower for stage I than for the higher stage tumors (Wilcoxon rank sum test, $p<0.001)$. As expected the two algorithms yielded significantly different sensitivity and specificity results $(p<0.001$, Fisher's exact test). For high risk adenomas the sensitivity was $14 \%$ (95\% CI, 3-63\%) vs $0 \%$ (95\% CI, 0-1.6\%), for the two algorithms. The positivity rates for adenomas were not different from the rates in the NED group, 18 \% (95\% CI, $12-25 \%$ ) vs $5 \%$ (95 \% CI, 2-9\%), for the two algorithms (Table 2).

The overall test specificity was $82 \%$ (95\% CI, 75-88 \%) vs. $95 \%$ (95\% CI, 91-98\%) for the two algorithms. To investigate if any subjects with NED falsely scored positive due to an occult cancer, all subsequent instances of cancer diagnoses three years after the initial colonoscopy were identified through hospital records. None of the subjects with NED that scored falsely positive were later diagnosed with cancer.

\section{Factors potentially affecting assay performance}

To investigate if the outcome of the Sept9 test was affected by any of the available demographic or clinical variables (excluding symptoms) the significance of all associations with Sept9 was tested using Fisher's exact test. Initially the continuous variable age was plotted against Sept9 outcome to look for trends of association, and to determine a cut-point for dichotomization of the age variable (Fig. 1). The plot revealed a tendency towards an increased false positive rate for NEDs with ages above $>65$ years, particularly for the high specificity $2 / 3$ algorithm (Fig. 1, right panel). After dichotomizing age at 65 years and testing for association to Sept 9 , the group with age $>65$ was significantly associated with increased false positive rates for both the $1 / 3$ and $2 / 3$ algorithms $(p=0.015$ and $p=0.05$ respectively). The analysis also revealed that age $>65$ was associated with an increased false negative rate 
Table 1 Demographic distribution of the nested cohort from the Endoscopy II prospective sample collection

\begin{tabular}{|c|c|c|c|c|c|}
\hline \multirow[t]{2}{*}{ Variable } & & \multirow{2}{*}{$\begin{array}{l}\text { Total } \\
299\end{array}$} & \multirow{2}{*}{$\begin{array}{l}\text { CRC } \\
128\end{array}$} & \multirow{2}{*}{$\begin{array}{l}\text { Adenoma } \\
21\end{array}$} & \multirow{2}{*}{$\begin{array}{l}\text { NED } \\
150 \\
\end{array}$} \\
\hline & & & & & \\
\hline \multirow[t]{2}{*}{ Sex } & Female & 151 & $65(51)$ & $11(52)$ & $75(50)$ \\
\hline & Male & 148 & $63(49)$ & $10(48)$ & $75(50)$ \\
\hline \multirow[t]{2}{*}{ Age } & $\leq 65$ & 183 & $74(58)$ & $11(52)$ & $98(65)$ \\
\hline & $>65$ & 116 & $54(42)$ & $10(48)$ & $52(35)$ \\
\hline \multirow[t]{2}{*}{ Rectal bleeding } & No & 166 & $54(42)$ & $10(48)$ & $102(68)$ \\
\hline & Yes & 133 & $74(58)$ & $11(52)$ & $48(32)$ \\
\hline \multirow[t]{2}{*}{ Anemia } & No & 250 & $102(80)$ & $19(90)$ & $129(86)$ \\
\hline & Yes & 49 & $26(20)$ & $2(10)$ & $21(14)$ \\
\hline \multirow[t]{2}{*}{ Weightloss } & No & 211 & $81(63)$ & $17(81)$ & $113(75)$ \\
\hline & Yes & 88 & $47(37)$ & $4(19)$ & $37(25)$ \\
\hline \multirow[t]{2}{*}{ Altered Defaecation } & No & 126 & $58(45)$ & $12(57)$ & $56(37)$ \\
\hline & Yes & 173 & $70(55)$ & $9(43)$ & $94(63)$ \\
\hline \multirow[t]{2}{*}{ Abdominal Pain } & No & 172 & $72(56)$ & $17(81)$ & $83(55)$ \\
\hline & Yes & 127 & $56(44)$ & $4(19)$ & $67(45)$ \\
\hline \multirow[t]{2}{*}{ Abdominal mass } & No & 283 & 119 (93) & 0 & $143(95)$ \\
\hline & Yes & 16 & $9(7)$ & 0 & $7(5)$ \\
\hline \multirow[t]{2}{*}{ Distention } & No & 241 & $103(80)$ & $21(100)$ & $117(78)$ \\
\hline & Yes & 58 & $25(20)$ & $0(0)$ & $33(22)$ \\
\hline \multirow[t]{2}{*}{ Hypertension } & No & 183 & $81(63)$ & $10(48)$ & $92(61)$ \\
\hline & Yes & 116 & $47(37)$ & $11(52)$ & $58(39)$ \\
\hline \multirow[t]{2}{*}{ Diabetes } & No & 280 & $117(91)$ & $16(76)$ & $147(98)$ \\
\hline & Yes & 19 & $11(9)$ & $5(24)$ & $3(2)$ \\
\hline \multirow[t]{2}{*}{ Arteriosclerosis } & No & 243 & $108(84)$ & $19(90)$ & $116(77)$ \\
\hline & Yes & 56 & $20(16)$ & $2(10)$ & $34(23)$ \\
\hline \multirow[t]{2}{*}{ Respiratory disease } & No & 267 & $117(91)$ & $21(100)$ & $129(86)$ \\
\hline & Yes & 32 & $11(9)$ & $0(0)$ & $21(14)$ \\
\hline \multirow[t]{2}{*}{ Arthritis } & No & 281 & $120(94)$ & $20(95)$ & $141(94)$ \\
\hline & Yes & 18 & $8(6)$ & $1(5)$ & $9(6)$ \\
\hline \multirow[t]{2}{*}{ Smoke\# } & No & 123 & $47(37)$ & $12(57)$ & $64(43)$ \\
\hline & Yes & & $81(63)$ & $9(43)$ & $86(57)$ \\
\hline \multirow[t]{2}{*}{ Alcohol\#\# } & Normal & 245 & $103(80)$ & $15(71)$ & $127(85)$ \\
\hline & Abuse & 54 & $25(20)$ & $6(29)$ & $23(15)$ \\
\hline \multirow[t]{4}{*}{ BMl\#\#\# } & $<18,5$ & 10 & $4(3)$ & $1(5)$ & $5(3)$ \\
\hline & 18,5 to 25 & 162 & $70(55)$ & $10(48)$ & $82(55)$ \\
\hline & 25 to 30 & 94 & $39(31)$ & $7(33)$ & $48(32)$ \\
\hline & $>30$ & 33 & $15(12)$ & $3(14)$ & $15(10)$ \\
\hline \multirow[t]{2}{*}{ Plasma volumea } & $<3.5 \mathrm{ml}$ & 160 & $70(55)$ & $6(29)$ & $84(56)$ \\
\hline & $3.5 \mathrm{ml}$ & 139 & $58(45)$ & $15(71)$ & $66(44)$ \\
\hline
\end{tabular}

Data are $\mathrm{n}(\%)$

\# Former smokers and current smokers pooled

\#\# Abuse: Women $>7$ units per week, Men $>14$ units per week

\#\#\# Underweight $<18,5$, Normal 18,5-25, Overweight 25-30, Heavy

overweight $>30$ a median $3.0 \mathrm{ml}$, range $(2-3.4 \mathrm{ml})$

NED No Evidence of Disease
Table 2 Summary of Epi proColon test performance in a nested case-control cohort from the Endoscopy II population

\begin{tabular}{lllll}
\hline 1/3 algorithm & & & \\
\hline Diagnosis & Subjects $(n)$ & Positive $(n)$ & Negative $(n)$ & Fraction $(95 \%$ Cl) \\
\hline CRC & 128 & 93 & 35 & $0.73(0.64-0.80)$ \\
stage I & 35 & 13 & 22 & $0.37(0.21-0.55)$ \\
stage II & 35 & 32 & 3 & $0.91(0.76-0.98)$ \\
stage III & 30 & 23 & 7 & $0.77(0.58-0.90)$ \\
stage IV & 28 & 25 & 3 & $0.89(0.72-0.98)$ \\
Non-CRC & 171 & 30 & 141 & $0.18(0.12-0.24)$ \\
Adenoma & 21 & 3 & 18 & $0.14(0.03-0.63)$ \\
NED & 150 & 27 & 123 & $0.18(0.12-0.25)$ \\
2/3 algorithm & & & \\
CRC & 128 & 75 & 53 & $0.59(0.50-0.67)$ \\
stage I & 35 & 6 & 29 & $0.17(0.07-0.34)$ \\
stage II & 35 & 26 & 9 & $0.74(0.57-0.88)$ \\
Stage III & 30 & 19 & 11 & $0.63(0.44-0.80)$ \\
Stage IV & 28 & 24 & 4 & $0.86(0.67-0.96)$ \\
Non-CRC & 171 & 7 & 164 & $0.04(0.02-0.08)$ \\
Adenoma & 21 & 0 & 21 & $0.00(0.00-0.16)$ \\
NED & 150 & 7 & 143 & $0.05(0.02-0.09)$ \\
\hline
\end{tabular}

\section{NED No Evidence of Disease}

Fraction: Positive fraction detected

Difference in $1 / 3$ vs $2 / 3$ algorithm: Fischer's Exact Test, $p<0.001$

Increasing proportion of true positive results with increasing tumor stage:

Wilcoxon rank sum test for trend, $z<0.001$

for the $2 / 3$ algorithm ( $p=0.007$, Tables 3 and 4). However, this significance was probably driven by an unintended imbalance in tumor stage distribution in the two age groups, with the $>65$ age group having significantly fewer stage III and IV tumors (Additional file 2: Table S2).

Of the other variables only Arthritis and Arteriosclerosis consistently affected Sept 9 outcome. Arthritis was associated with an increased false negative rate (Table 3 ), which was significant for the $1 / 3$ algorithm $(p=0.005)$ and borderline for the $2 / 3$ algorithm $(p=0.07)$. Arteriosclerosis was associated with an increased false positive rate (Table 4). This association was significant for the $2 /$ 3 algorithm $(p=0.007)$ and borderline for the $1 / 3$ algorithm $(p=0.07)$.

Female gender was associated with an increased false negative rate for the more sensitive $1 / 3$ algorithm but not the more specific $2 / 3$ algorithm.

As a low sample input means less cfDNA available for analysis, it was tested whether a low plasma volume had any effect on Sept9 performance. No effect on sensitivity was observed (Fishers exact test $p=0.69$ vs $p=0.59$ for the two algorithms). On the opposite, a low plasma volume surprisingly seemed to produce more false positive 
1/3 Algorithm

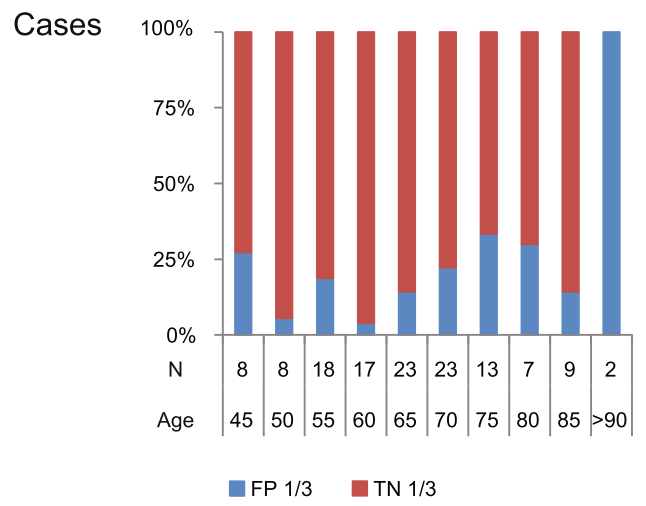

Controls $100 \%$

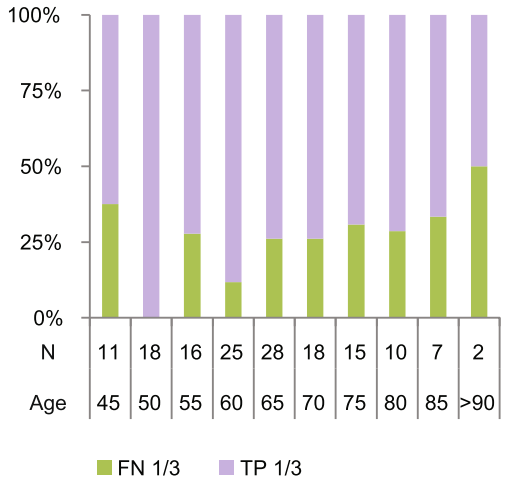

2/3 Algorithm
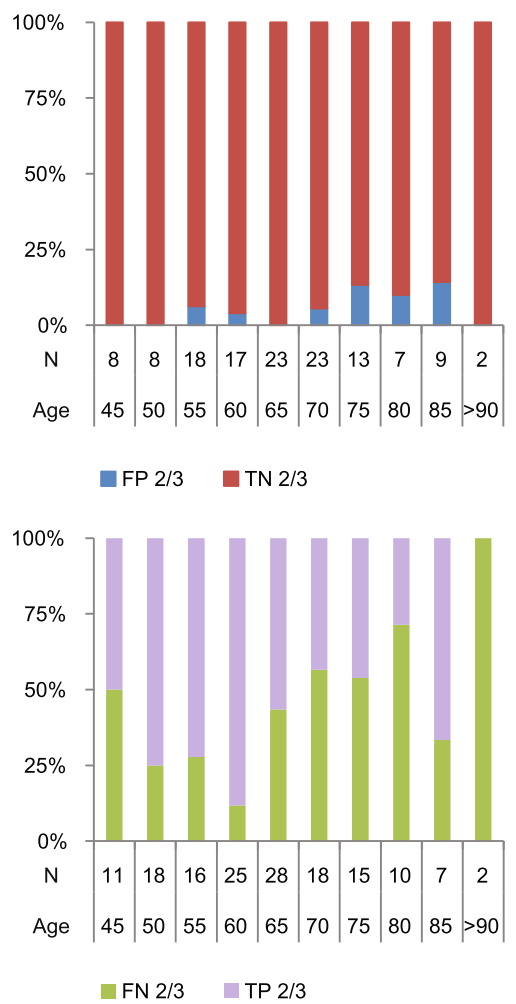

Fig. 1 Age plotted against Sept9 outcome. FP: False positive, TN: True negative, FN: False negative, TP: True positive. N: Number of subjects in each category. Age: All ages younger or equal to the age interval mentioned. $-1 / 3$ and $2 / 3$ refers to the PCR-algorithms used

controls (Table 4). However, this significance was probably driven by age, as more subjects aged $>65$ had lower plasma volumes (Additional file 3: Table S3).

\section{Sept9 as predictor of CRC}

Logistic regression models were built to evaluate: i) how well a positive Sept9 test predicts CRC, ii) which, if any, of the available exposure variables might modify Sept9's ability to predict CRC, and iii) the strength of the association between the Sept 9 test and the diagnosis of CRC, when taking these variables into account.

First, it was evaluated which of the available variables (including symptoms) were associated with CRC. In univariate models, only Sept9 (OR 8.25, 95 \% CI 4.8314.09, $p<0.001$ ), rectal bleeding (OR $2.8295 \% \mathrm{CI}$ $1.76-4.5, p<0.001$ ) and Diabetes (OR 5.89, $95 \%$ CI 1.68-20.68, $p=0.006$ ) showed a significant association (Additional file 4: Table S4).

To identify variables associated with Sept9 outcome, univariate logistic regression models were built with Sept 9 as outcome variable and all other variables consecutively as explaining variable. Diabetes was significant with an OR of 5.2 (95\% CI 1.4-19.1), and likewise was age when adjusted for tumor stage (OR 2.06, 95 \% CI 1.1-3.8), but none of the other variables (Additional file 5: Table S5).
Next, we checked if any variables modified the outcome of the Sept 9 test. Age $>65$ and Arthritis was found to be significant modifiers with an OR of 2.46 (95 \% CI 1.14-5.30) and 0.03 (95\% CI 0.00-0.22), respectively. Consequently, we allowed for effect modification from these factors in the final multivariate regression model (Additional file 6: Table S6).

All variables found to be associated with Sept9 by either Fishers exact test or regression (age, Arthritis, Arteriosclerosis, and Diabetes) were included in a final multivariate regression model with CRC as outcome. Interestingly, the adjusted OR associated with a positive Sept9 test increased from 8.25 to 29.46 (95\% CI $12.58-69.02, p<0.001)$ for the $1 / 3$ algorithm Table 5. Similar results were obtained for the $2 / 3$ algorithm (Additional file 7: Table S7).

\section{Discussion}

\section{Clinical performance}

It is well examined that the Sept9 test can be used to identify occult CRC. More than 15.000 subjects have been tested and the reported sensitivity ranges from 36.6 to $95.6 \%$ [14]. The test has primarily been applied to cases and controls selected from separate populations i.e. cases were typically patients with symptomatic CRC 
Table 3 Sept9 positivity of individuals with CRC

\begin{tabular}{|c|c|c|c|c|c|c|c|c|c|}
\hline \multirow{2}{*}{ Variable } & & \multicolumn{4}{|c|}{ 1/3 algorithm } & \multicolumn{4}{|c|}{ 2/3 algorithm } \\
\hline & & $\begin{array}{l}P \\
93\end{array}$ & $\begin{array}{l}N \\
35\end{array}$ & $\begin{array}{l}\%(95 \% \mathrm{Cl}) \\
73\end{array}$ & $\begin{array}{l}p^{*} \\
N R\end{array}$ & $\begin{array}{l}P \\
75\end{array}$ & $\begin{array}{l}N \\
53\end{array}$ & $\begin{array}{l}\%(95 \% \mathrm{Cl}) \\
59\end{array}$ & $\begin{array}{l}p^{*} \\
N R\end{array}$ \\
\hline \multirow[t]{2}{*}{ Sex } & Female & 42 & 23 & $65(52-76)$ & 0.05 & 34 & 31 & $52(40-65)$ & 0.16 \\
\hline & Male & 51 & 12 & $81(69-90)$ & & 41 & 22 & 65 ( 52-77) & \\
\hline \multirow[t]{2}{*}{ Age } & $\leq 65$ & 57 & 17 & $77(66-86)$ & 0.23 & 51 & 23 & $69(59-81)$ & 0.007 \\
\hline & $>65$ & 36 & 18 & $67(53-79)$ & & 24 & 30 & $44(31-59)$ & \\
\hline \multirow[t]{2}{*}{ Hypertension } & No & 60 & 21 & $74(63-83)$ & 0.68 & 49 & 32 & $60(49-71)$ & 0.58 \\
\hline & Yes & 33 & 14 & $70(55-83)$ & & 26 & 21 & $55(40-70)$ & \\
\hline \multirow[t]{2}{*}{ Diabetes } & No & 84 & 33 & $72(63-80)$ & 0.73 & 67 & 50 & $57(48-66)$ & 0.36 \\
\hline & Yes & 9 & 2 & $82(48-98)$ & & 8 & 3 & 73 (39-94) & \\
\hline \multirow[t]{2}{*}{ Arteriosclerosis } & No & 80 & 28 & $74(65-82)$ & 0.42 & 65 & 43 & $60(50-69)$ & 0.46 \\
\hline & Yes & 13 & 7 & $65(41-85)$ & & 10 & 10 & $50(27-73)$ & \\
\hline \multirow[t]{2}{*}{ Respiratory disease } & No & 87 & 30 & $74(65-82)$ & 0.17 & 71 & 46 & $61(51-70)$ & 0.20 \\
\hline & Yes & 6 & 5 & $55(23-83)$ & & 4 & 7 & $36(11-69)$ & \\
\hline \multirow[t]{2}{*}{ Arthritis } & No & 91 & 29 & $76(61-83)$ & 0.005 & 73 & 47 & $61(52-70)$ & 0.07 \\
\hline & Yes & 2 & 6 & $25(3-65)$ & & 2 & 6 & $25(3-65)$ & \\
\hline \multirow[t]{2}{*}{ Smoke\# } & No & 38 & 9 & 81 (67-91) & 0.08 & 29 & 18 & $62(46-75)$ & 0.36 \\
\hline & Yes & 55 & 26 & $68(57-78)$ & & 46 & 35 & $57(45-68)$ & \\
\hline \multirow[t]{2}{*}{ Alcohol\#\# } & Normal use & 78 & 25 & $76(66-84)$ & 0.14 & 64 & 39 & $62(52-72)$ & 0.12 \\
\hline & Abuse & 15 & 10 & $60(39-79)$ & & 11 & 14 & $44(24-65)$ & \\
\hline \multirow[t]{4}{*}{ BMI } & $<18,5$ & 3 & 1 & 75 (19-99) & 0.97 & 3 & 1 & 75 (19-99) & 0.95 \\
\hline & 18,5 to 25 & 52 & 18 & $74(62-84)$ & & 41 & 29 & $59(46-70)$ & \\
\hline & 25 to 30 & 27 & 12 & $69(52-83)$ & & 22 & 17 & $56(40-72)$ & \\
\hline & $>30$ & 11 & 4 & $73(45-92)$ & & 9 & 6 & $60(32-84)$ & \\
\hline \multirow[t]{3}{*}{ Tumor site } & Left & 23 & 7 & $77(58-90)$ & 0.67 & 17 & 13 & $57(37-75)$ & 0.70 \\
\hline & Right & 37 & 17 & $69(54-80)$ & & 34 & 20 & $63(49-76)$ & \\
\hline & Rectum & 33 & 11 & $75(60-87)$ & & 24 & 20 & 55 (39-70) & \\
\hline \multirow[t]{2}{*}{ MSIa } & Stable & 35 & 15 & $70(55-82)$ & 1.00 & 28 & 22 & $56(41-70)$ & 1.00 \\
\hline & Unstable & 8 & 3 & 73 (39-94) & & 6 & 5 & $55(23-83)$ & \\
\hline \multirow[t]{2}{*}{ Plasma volume } & $<3.5 \mathrm{ml}$ & 52 & 18 & $74(62-84)$ & 0.69 & 43 & 27 & 61 (49-73) & 0.59 \\
\hline & $3.5 \mathrm{ml}$ & 41 & 17 & $71(57-82)$ & & 32 & 26 & $55(42-68)$ & \\
\hline
\end{tabular}

${ }^{*} p$-value, two-sided Fisher's exact test, $p<0.05$ considered statistically significant $N R$ Not relevant, $P$ Positive, $N$ Negative, $\%$ Positive fraction detected

\#Former smokers and smokers pooled

\#\#Abuse: Women > 7 units per week, Men >14 units per week

aMSI determined by Immunohistochemistry, data not available on all cases

found at colonoscopy, while controls were often screening subjects or symptomatic individuals found to be tumor negative after colonoscopy. This makes statistical comparison uncertain, and furthermore does not fulfil the REMARK criteria [15]. The present study, with cases and controls selected from the same cohort of symptomatic subjects, who all had their blood samples drawn and processed according to the same standard operating procedure, fulfills the requirement of the REMARK criteria. Moreover the included subjects have gender and comorbidity distributions similar to what can be expected in a screening population. This might be the reason that the overall results are more similar to that of recent screening based cohorts than of earlier case-control studies $[10,12,13]$. The present study indicated that the Sept9 assay had low sensitivity in detecting early stage tumors (adenomas and stage I carcinomas). However, that may potentially be explained by the limited plasma volume used for analysis $(\leq 3.5 \mathrm{ml})$. It has been reported that the number of ctDNA (cell-free tumor DNA) genome equivalents per 5 milliliter blood often is less than ten for patients with stage I carcinomas [16]. Accordingly, to 
Table 4 Sept9 positivity of individuals with NED

\begin{tabular}{|c|c|c|c|c|c|c|c|c|c|}
\hline \multirow{2}{*}{ Variable } & & \multicolumn{4}{|c|}{ 1/3 algorithm } & \multicolumn{4}{|c|}{ 2/3 algorithm } \\
\hline & & $\begin{array}{l}P \\
27\end{array}$ & $\begin{array}{l} \\
123 \\
\end{array}$ & $\begin{array}{l}\%(95 \% \mathrm{Cl}) \\
18\end{array}$ & $\begin{array}{l}p^{*} \\
N R\end{array}$ & $\begin{array}{l}P \\
27\end{array}$ & $\begin{array}{l}N \\
123\end{array}$ & $\begin{array}{l}\%(95 \% \mathrm{Cl}) \\
18\end{array}$ & $\begin{array}{l}p^{*} \\
\mathrm{NR}\end{array}$ \\
\hline \multirow[t]{2}{*}{ Sex } & Female & 11 & 64 & $15(8-25)$ & 0.4 & 2 & 73 & $3(0-9)$ & 0.44 \\
\hline & Male & 16 & 59 & $21(13-32)$ & & 5 & 70 & $7(2-15)$ & \\
\hline \multirow[t]{2}{*}{ Age } & $\leq 65$ & 12 & 86 & $12(6-20)$ & 0.015 & 2 & 96 & $2(0-7)$ & 0.05 \\
\hline & $>65$ & 15 & 37 & $29(17-43)$ & & 5 & 47 & $10(3-21)$ & \\
\hline \multirow[t]{2}{*}{ Hypertension } & No & 18 & 74 & $20(12-29)$ & 0.66 & 4 & 88 & $4(1-11)$ & 1.00 \\
\hline & Yes & 9 & 49 & $16(7-27)$ & & 3 & 55 & $5(1-14)$ & \\
\hline \multirow[t]{2}{*}{ Diabetes } & No & 25 & 122 & $17(11-24)$ & 0.08 & 6 & 141 & $4(2-9)$ & 0.13 \\
\hline & Yes & 2 & 1 & 67 (9-99) & & 1 & 2 & $33(0-91)$ & \\
\hline \multirow[t]{2}{*}{ Arteriosclerosis } & No & 17 & 99 & $15(9-22)$ & 0.07 & 2 & 114 & $2(0-6)$ & 0.007 \\
\hline & Yes & 10 & 24 & $29(12-39)$ & & 5 & 29 & $15(5-31)$ & \\
\hline \multirow[t]{2}{*}{ Respiratory disease } & No & 24 & 105 & $19(12-26)$ & 0.77 & 5 & 124 & $4(1-8)$ & 0.25 \\
\hline & Yes & 3 & 18 & $14(3-36)$ & & 2 & 19 & $10(1-30)$ & \\
\hline \multirow[t]{2}{*}{ Arthritis } & No & 23 & 118 & $16(11-23)$ & 0.06 & 7 & 134 & $5(2-10)$ & 1.00 \\
\hline & Yes & 4 & 5 & $44(14-79)$ & & 0 & 9 & $0(0-34)$ & \\
\hline \multirow[t]{2}{*}{ Smoke\# } & No & 10 & 54 & $16(8-27)$ & 0.33 & 2 & 62 & $3(0-11)$ & 0.36 \\
\hline & Yes & 17 & 69 & $20(12-30)$ & & 5 & 81 & $6(2-13)$ & \\
\hline \multirow[t]{2}{*}{ Alcohol\#\# } & Normal use & 24 & 103 & $19(12-27)$ & 0.77 & 6 & 121 & $5(2-10)$ & 1.00 \\
\hline & Abuse & 3 & 20 & $13(3-34)$ & & 1 & 22 & $4(0-22)$ & \\
\hline \multirow[t]{4}{*}{ BMI } & $<18,5$ & 0 & 5 & $0(0-52)$ & 0.92 & 0 & 5 & $0(0-52)$ & 0.61 \\
\hline & 18,5 to 25 & 15 & 67 & $18(11-28)$ & & 5 & 77 & $6(2-14)$ & \\
\hline & 25 to 30 & 9 & 39 & $19(9-33)$ & & 1 & 47 & $2(0-11)$ & \\
\hline & $>30$ & 3 & 12 & $20(4-48)$ & & 1 & 14 & $7(0-32)$ & \\
\hline \multirow[t]{2}{*}{ Plasma volume } & $<3.5 \mathrm{ml}$ & 20 & 64 & $24(15-34)$ & 0.05 & 6 & 78 & 7 (3-15) & 0.14 \\
\hline & $3.5 \mathrm{ml}$ & 7 & 59 & $11(4-21)$ & & 1 & 65 & $2(0-8)$ & \\
\hline
\end{tabular}

${ }^{*} p$-value, two-sided Fisher's exact test, $p<0.05$ considered statistically significant

NR Not relevant, $P$ Positive, $N$ Negative, \% Positive fraction, NED No Evidence of Disease

\#Former smokers and current smokers pooled

\#\#Abuse: Women > 7 units per week, Men >14 units per week

Table 5 Predictors of Colorectal Cancer, 1/3 algorithm

\begin{tabular}{|c|c|c|}
\hline Predictor & OR $(95 \% \mathrm{Cl})$ & $p$-value ${ }^{*}$ \\
\hline Sept9 -crude estimate & $8.25(4.83-14.09)$ & 0.000 \\
\hline Correction for other variables & Multivariate OR (95 \% Cl) & $p$-value* \\
\hline Sept9 -adjusted OR & $29.46(12.58-69.02)$ & 0.000 \\
\hline \multicolumn{3}{|l|}{ Demographic characteristics } \\
\hline Age $>65$ & $2.80(1.23-6.36)$ & 0.014 \\
\hline Age as effect-modificator of Sept9 & $0.24(0.07-0.80)$ & 0.020 \\
\hline \multicolumn{3}{|l|}{ Co-morbidities } \\
\hline Diabetes & $2.26(0.51-10.05)$ & 0.283 \\
\hline Arterioschlerosis & $0.39(0.18-0.86)$ & 0.019 \\
\hline Arthritis & $4.88(1.30-18.42)$ & 0.019 \\
\hline Arthritis as effect-modificator of Sept9 & $0.02(0.000 .22)$ & 0.001 \\
\hline
\end{tabular}

${ }^{*} p<0.05$ considered statistically significant 
increase the sensitivity towards early stage tumors it will probably be necessary to increase the plasma volume. In addition to plasma volume other factors may potentially also influence adenoma sensitivity. A recent report indicated that the methylation of the Sept9 locus is a late event in the transformation of adenomas to carcinomas [17]; even if adenomas release ctDNA, it may not be methylated and hence may not be detected by the Sept 9 assay. One way to mitigate this particular problem could be to use multiple markers, including markers targeting adenomas, rather than Sept9 alone. Along these lines we hypothesize that to reach optimal sensitivity and specificity of both adenomas and early stage carcinomas an increased plasma volume and a multiplex test, targeting several colorectal neoplasia specific methylation markers, is needed.

\section{Factors with impact on assay performance}

The influence of demographic parameters on the Sept9 test has previously only been sparsely examined. In line with other reports we showed that gender and tumor localization did not affect assay sensitivity [10, 12, 13, 18, 19]. Previously, deregulation of Sept9 expression has been reported to be associated with genomic instability by at least two mechanisms associated to chromosomal instability (CIN), namely by mitotic spindle defects and/ or incomplete cell division [20]. Therefore we investigated whether Sept9 methylation was better at predicting CIN than microsatellite unstable (MSI) tumors? Surprisingly, we could not identify differences between the positivity rates for MSI and microsatellite stable (MSS) cancers (Fishers exact test, $p=1.00$, Table 3).

The only factor besides tumor stage recurrently reported to influence Sept9 performance is age. Higher age has been described to be associated with both decreasing sensitivity and specificity $[12,13]$. Our findings are in support of this observation, as we also showed the assay to have reduced specificity for the oldest test subjects (age > 65). The decreasing specificity with older age might be partly explained by the known correlation between chronological age and increased genome-wide DNA methylation changes [21], but could also be due to a higher prevalence of chronic diseases in elderly compared to younger subjects. Several studies associate various chronic diseases with DNA methylation changes $[22,23]$. This might lead to a higher risk of coincident and non-CRC related methylation of the Sept 9 locus in elderly subjects. Though a positive Sept 9 test should not be regarded as confirmative evidence for CRC, and should always be confirmed by a colonoscopy, a decreased specificity with age $>65$ challenges a test aimed at subjects at age $50-75$ years, and lead to larger down-stream costs. To address this problem an age-differentiated use of the two Sept 9 scoring algorithms could be considered: if the $1 / 3$ algorithm is applied to subjects $\leq 65$ and the $2 / 3$ algorithm is applied to subjects $>65$ years the combined sensitivity and specificity is $64 \%$ and $89 \%$ compared to $0.73 \%$ and $0.82 \%$ for the $1 / 3$ algorithm alone (Additional file 8 : Table S8).

In contrast to earlier studies, several co-morbidities influenced the Sept9 test in the present study [10, 13, 24]. Particularly, subjects with Arthritis were difficult to score correctly for the Sept 9 test. This has not been reported earlier. Nevertheless, in 2008 the assay was tested in a cohort of 315 control subjects without CRC, but with different comorbidities [24]. By going through the reported data we observed, consistent with the present study, that $20 \%$ of NED subjects with Rheumatoid arthritis were false positive and similarly that patients with Lupus also had a high false positive rate (14.2\%). Since 1966 it has been known that autoimmune inflammatory diseases such as Lupus, Polyarthritis, or Rheumatoid arthritis are associated with significant elevated levels of cfDNA [25-27]. We therefore speculate that the decreased assay sensitivity observed in subjects with Arthritis could be due to increased circulating levels of arthritis-associated cfDNA, making it difficult to detect the few copies of methylated ctDNA from the CRC. Further, a recently published study of DNA methylome changes in Rheumatoid arthritis indicates that DNA hypermethylation is a part of the disease etiology and that the methylation alterations continue to evolve as the disease progresses to chronic Rheumatoid arthritis. We consider that this dynamic pattern may lead to cancer-independent methylation of Sept 9 , and hence a higher false positivity rate among subjects with NED and arthritis [28].

For subjects with NED, Diabetes and Arteriosclerosis showed borderline significant association to false positive Sept9 results. Both diseases generate generalized inflammation in the body, and hence potentially increased levels of cfDNA and methylome changes, however additional studies are needed to establish this confidently.

\section{Strengths and limitations of our study}

A major strength of this study is that it is based on a well-characterized nested case-control design, which minimizes the risk of the selection bias that was seen in several of the early Sept9 studies. The available lifestyle factors were self-reported by the patients, with the uncertainty this may cause, whereas BMI, follow-up and information about chronic diseases were collected from the medical records. In order to eliminate potential confounding effects from age and gender we matched cases and controls on these parameters. The male and female cases were further matched on tumor site and stage. Collectively this fulfills the requirements for statistical 
comparison of case and controls. One obvious limitation of the study is the size of the cohort, which counted only 299 subjects. Accordingly, near-significant differences between cases and controls (Type II error) may still reflect potentially interesting observations. Another limitation is that the Sept9 test is validated for $3.5 \mathrm{~mL}$ of plasma and only 139 subjects fulfilled this requirement. Though no significant difference was observed as a result of the lower plasma volume, this could influence especially the overall assay sensitivity. Finally, we allowed for a wide age range in our cohort with 45 subjects $<50$ years of age. Therefore the age of the cohort differs slightly from that of a screening cohort, where all subjects are $>50$. The wide age range may enhance the differences in assay performance due to age when subjects $>65$ are compared to subjects $\leq 65$.

\section{Conclusions}

In conclusion, the present nested case-control study indicates that the Sept 9 assay has an overall sensitivity of $73 \%$ and a specificity of $82 \%$ (1/3 algorithm). While these numbers appear promising, the sensitivity for adenomas and stage I tumors was limited. Naturally, the utility of the assay for CRC population screening will require improved sensitivity for detection of these early stage tumors. We consider that increasing the plasma volume will be essential to achieve the needed improvement, but this must be tested in future studies. In addition, we showed that high age and comorbidities like Arthritis, Arteriosclerosis, and Diabetes affected assay performance negatively. Taken together this might partly explain why the performance of the Sept9 assay in recent screening based studies varies from the performance estimates of previous retrospective case-control studies. In addition, the findings indicate that age and comorbidities alter both the DNA methylome and the levels of circulating DNA in an individual. This implies that all future blood-based assays, targeting a few ctDNA copies in a large pool of cfDNA, especially methylationsensitive assays, may be affected.

\section{Additional files}

Additional file 1: Table S1. Association between variables in cohort *Two-sided Fisher's exact test, all numbers are $p$-values. $p<0.05$ considered statistically significant. \# Former smokers and current smokers pooled vs non-smokers. \#\# Abuse: Women $>7$ units per week, Men > 14 units per week. \#\#\# Underweight < 18,5, Normal 18,5-25, Overweight 25-30, Heavy overweight $>30$. (DOC $35 \mathrm{~kb}$ )

Additional file 2: Table S2. Individuals with CRC stratified by tumorstage and age. $p$-value $<0.001$ Fishers exact test. (DOC $30 \mathrm{~kb}$ )

Additional file 3: Table S3. Positivity of subjects with NED stratified by plasma volume and age. * Two-sided Fisher's exact test, $p<0.05$ considered statistically significant Fraction: Positive fraction detected. NR: Not Relevant. NED: No Evidence of Disease. (DOC 35 kb)

Additional file 4: Table S4. Predictors of CRC in univariate regression, 1/3 algorithm. a p-values for Sept9 2/3 algorithm similar (data not shown). ${ }^{*} p$-value $<0.05$ is considered statistically significant. \# Former smokers and current smokers pooled vs non-smokers. \#\# Abuse: Women $>7$ units per week, Men >14 units per week. \#\#\# Underweight < 18,5, Normal 18,5-25, Overweight 25-30, Heavy overweight $>30$. (DOC $39 \mathrm{~kb}$ )

Additional file 5: Table S5. Factors associated with a positive Sept9 outcome. a $p$-values for Sept9 2/3 algorithm similar (data not shown). * $p$-value $<0.05$ is considered statistically significant. \# Former smokers and current smokers pooled vs non-smokers. \#\# Abuse: Women $>7$ units per week, Men >14 units per week. \#\#\# Underweight < 18,5, Normal 18,5-25, Overweight 25-30, Heavy overweight >30. (DOC 33 kb)

Additional file 6: Table S6. Effect modificators of Sept9 positivity in CRC. a $p$-values for Sept9 2/3 algorithm similar (data not shown). ${ }^{*} p$-value $<0.05$ is considered statistically significant. \# Former smokers and current smokers pooled vs non-smokers. \#\# Abuse: Women $>7$ units per week, Men $>14$ units per week. \#\#\# Underweight $<18,5$, Normal 18,5-25, Overweight 25-30, Heavy overweight >30. (DOC 34 kb)

Additional file 7: Table S7. Predictors of Colorectal Cancer, 2/3 algorithm. ${ }^{*} p<0.05$ considered statistically significant. (DOC $37 \mathrm{~kb}$ )

Additional file 8: Table S8. Sensitivity and specificity for Sept9 after age adjusted combinations of positivity algorithms. (DOC $31 \mathrm{~kb}$ )

\section{Abbreviations}

CRC: Colorectal cancer; DNA: Deoxyribo-nucleic-acid; PCR: Polymerase chain reaction; Cl: Confidence interval; OR: Odds ratio; FOBT/FIT: Fecal occult blood test/ Fecal immunochemical test; RNA: Ribo-nucleic-acid; HNPCC: Hereditary nonpolyposis colorectal cancer/Lynch syndrome; FAP: Familial adenomatous polyposis; TNM: Classification system for malignant tumors; DCCG: Danish colorectal cancer group; AMI: Acute myocardial infarction; NED: No evidence of disease; UICC: Union for international cancer control; EDTA: Ethylenediamine-tetraacetic-acid; cfDNA: Circulating cell-free DNA; ACTB: Actin beta, protein coding gene used as internal control in this setting; Ct: Cycle threshold in PCR reactions; REMARK: REporting recommendations for tumour MARKer prognostic studies, article; ctDNA: Circulating cell-free tumor DNA; CIN: Chromosomal instability; MSI: Microsatellite unstable; MSS: Microsatellite stable.

\section{Competing interest}

The authors declare that they have no competing interests.

\section{Authors' contributions}

MWO, HJN, TFO and CLA contributed to the study design. HJN collected and managed the Endoscopy II cohort. MWO and CLA performed statistical analyses. MWO drafted the manuscript. TFO provided constructive critical input. HJN, TFO and CLA revised the manuscript. All authors approved of the final manuscript.

\section{Authors' informations}

HJN participated on behalf of the Danish Study Group on Early Detection of Colorectal Cancer:

Lars N. Jørgensen, MD, DMSc, Department of Surgical Gastroenterology, Bispebjerg Hospital, Copenhagen,

Mogens R. Madsen, MD, Department of Surgery, Herning Hospital, Herning, Jesper Vilandt, MD, Department of Surgery, Hillerød Hospital, Hillerød, Thore Hillig, MSc, Ph.D., Department of Clinical Biochemistry, Hillerød Hospital, Hillerød,

Michael Klærke, MD, Department of Surgery, Horsens Hospital, Horsens, Jens Andersen, MD, Department of Surgical Gastroenterology, Hvidovre Hospital, Hvidovre

Knud T. Nielsen, MD, Department of Surgery, Randers Hospital, Randers, Søren Laurberg, MD, DMSc, Department of Surgical Gastroenterology, Aarhus Hospital THG, Aarhus

\section{Acknowledgements}

We would like to thank all the subjects who agreed to participate in the Endoscopy II cohort. We would also like to thank research nurses, technicians and secretaries at the collaborating hospitals and laboratories for their skillful work and major engagement in the entire project. Finally we wish to thank Epigenomics $\mathrm{GmbH}$ Berlin, for skillfully processing the plasma samples and running the Sept9 assay (EpiproColon). 


\section{Author details}

'Department of Molecular Medicine, MOMA, Aarhus University Hospital, Skejby DK-8200Aarhus N, Denmark. ${ }^{2}$ Department of Surgical Gastroenterology 360, Hvidovre Hospital, University of Copenhagen, DK-2650 Hvidovre, Denmark.

Received: 14 July 2015 Accepted: 16 October 2015

Published online: 29 October 2015

\section{References}

1. GLOBOCAN 2012 v1.0, Cancer Incidence and Mortality Worldwide: IARC CancerBase No. 11 [database on the Internet]. International Agency for Research on Cancer. 2012. Available from: http://globocan.iarc.fr. Accessed: 31/05/2015

2. Bjerregaard NC, Tottrup A, Sorensen HT, Laurberg S. Diagnostic value of self-reported symptoms in Danish outpatients referred with symptoms consistent with colorectal cancer. Colorectal Dis. 2007;9(5):443-51. doi:10.1111/j.1463-1318.2006.01170.x.

3. Mandel JS, Bond JH, Church TR, Snover DC, Bradley GM, Schuman LM, et al. Reducing mortality from colorectal cancer by screening for fecal occult blood. Minnesota Colon Cancer Control Study. N Engl J Med. 1993:328(19):1365-71. doi:10.1056/NEJM199305133281901.

4. Pande R, Froggatt P, Baragwanath P, Harmston C. Survival outcome of patients with screening versus symptomatically detected colorectal cancers. Colorectal Dis. 2013;15(1):74-9. doi:10.1111/j.1463-1318.2012.03120.x.

5. Heichman KA. Blood-based testing for colorectal cancer screening. Mol Diagn Ther. 2014;18(2):127-35. doi:10.1007/s40291-013-0074-z.

6. Nielsen HJ, Jakobsen KV, Christensen IJ, Brunner N, Danish Study Group on Early Detection of Colorectal C. Screening for colorectal cancer: possible improvements by risk assessment evaluation? Scand J Gastroenterol. 2011:46(11):1283-94. doi:10.3109/00365521.2011.610002.

7. Regueiro CR, Committee AGAFT. AGA Future Trends Committee report: Colorectal cancer: a qualitative review of emerging screening and diagnostic technologies. Gastroenterology. 2005;129(3):1083-103. doi:10.1053/j.gastro.2005.06.012

8. Ladabaum U, Allen J, Wandell M, Ramsey S. Colorectal cancer screening with blood-based biomarkers: cost-effectiveness of methylated septin 9 DNA versus current strategies. Cancer Epidemiol Biomarkers Prev. 2013;22(9):1567-76. doi:10.1158/1055-9965.EPI-13-0204.

9. deVos T, Tetzner R, Model F, Weiss G, Schuster M, Distler J, et al. Circulating methylated SEPT9 DNA in plasma is a biomarker for colorectal cancer. Clin Chem. 2009;55(7):1337-46. doi:10.1373/clinchem.2008.115808.

10. Johnson DA, Barclay RL, Mergener K, Weiss G, Konig T, Beck J, et al. Plasma Septin9 versus fecal immunochemical testing for colorectal cancer screening: a prospective multicenter study. PLoS One. 2014;9(6), e98238. doi:10.1371/journal.pone.0098238

11. Lofton-Day C, Model F, Devos T, Tetzner R, Distler J, Schuster M, et al. DNA methylation biomarkers for blood-based colorectal cancer screening. Clin Chem. 2008;54(2):414-23. doi:10.1373/clinchem.2007.095992.

12. Church TR, Wandell M, Lofton-Day C, Mongin SJ, Burger M, Payne SR, et al. Prospective evaluation of methylated SEPT9 in plasma for detection of asymptomatic colorectal cancer. Gut. 2014;63(2):317-25. doi:10.1136/gutjnl-2012-304149.

13. Potter NT, Hurban P, White MN, Whitlock KD, Lofton-Day CE, Tetzner R, et al. Validation of a real-time PCR-based qualitative assay for the detection of methylated SEPT9 DNA in human plasma. Clin Chem. 2014;60(9):1183-91. doi:10.1373/clinchem.2013.221044.

14. Li Y, Song L, Gong Y, He B. Detection of colorectal cancer by DNA methylation biomarker SEPT9: past, present and future. Biomark Med. 2014;8(5):755-69. doi:10.2217/bmm.14.8.

15. McShane LM, Altman DG, Sauerbrei W, Taube SE, Gion M, Clark GM, et al. REporting recommendations for tumour MARKer prognostic studies (REMARK). Br J Cancer. 2005;93(4):387-91. doi:10.1038/sj.bjc.6602678.

16. Bettegowda C, Sausen M, Leary RJ, Kinde I, Wang Y, Agrawal N, et al. Detection of circulating tumor DNA in early- and late-stage human malignancies. Sci Transl Med. 2014;6((224):224ra24. doi:10.1126/ scitranslmed.3007094.

17. Wasserkort R, Kalmar A, Valcz G, Spisak S, Krispin M, Toth K, et al. Aberrant septin 9 DNA methylation in colorectal cancer is restricted to a single CpG island. BMC Cancer. 2013;13:398. doi:10.1186/1471-2407-13-398.
18. Ahlquist DA, Taylor WR, Mahoney DW, Zou H, Domanico M, Thibodeau SN, et al. The stool DNA test is more accurate than the plasma septin 9 test in detecting colorectal neoplasia. Clin Gastroenterol Hepatol. 2012;10(3):272-7. doi:10.1016/j.cgh.2011.10.008. e1.

19. Toth K, Wasserkort R, Sipos F, Kalmar A, Wichmann B, Leiszter K, et al. Detection of methylated septin 9 in tissue and plasma of colorectal patients with neoplasia and the relationship to the amount of circulating cell-free DNA. PLoS One. 2014;9(12), e115415. doi:10.1371/journal.pone.0115415.

20. Peterson EA, Stanbery L, Li C, Kocak H, Makarova O, Petty EM. SEPT9_i1 and genomic instability: mechanistic insights and relevance to tumorigenesis. Genes Chromosomes Cancer. 2011;50(11):940-9. doi:10.1002/gcc.20916.

21. Fraga MF, Ballestar E, Paz MF, Ropero S, Setien F, Ballestar ML, et al. Epigenetic differences arise during the lifetime of monozygotic twins. Proc Natl Acad Sci U S A. 2005;102(30):10604-9. doi:10.1073/pnas.0500398102.

22. Handy DE, Castro R, Loscalzo J. Epigenetic modifications: basic mechanisms and role in cardiovascular disease. Circulation. 2011;123(19):2145-56. doi:10.1161/CIRCULATIONAHA.110.956839.

23. Keating ST, El-Osta A. Epigenetics and metabolism. Circ Res. 2015;116(4):715-36. doi:10.1161/CIRCRESAHA.116.303936.

24. Grutzmann R, Molnar B, Pilarsky C, Habermann JK, Schlag PM, Saeger HD, et al. Sensitive detection of colorectal cancer in peripheral blood by septin 9 DNA methylation assay. PLoS One. 2008;3(11), e3759. doi:10.1371/ journal.pone.0003759.

25. Bartoloni E, Ludovini V, Alunno A, Pistola L, Bistoni O, Crino L, et al. Increased levels of circulating DNA in patients with systemic autoimmune diseases: A possible marker of disease activity in Sjogren's syndrome. Lupus. 2011;20(9):928-35. doi:10.1177/0961203311399606.

26. Tan EM, Schur PH, Carr RI, Kunkel HG. Deoxybonucleic acid (DNA) and antibodies to DNA in the serum of patients with systemic lupus erythematosus. J Clin Invest. 1966;45(11):1732-40. doi:10.1172/JCl105479.

27. Zhong XY, von Muhlenen I, Li Y, Kang A, Gupta AK, Tyndall A, et al. Increased concentrations of antibody-bound circulatory cell-free DNA in rheumatoid arthritis. Clin Chem. 2007:53(9):1609-14. doi:10.1373/ clinchem.2006.084509.

28. Ai R, Whitaker JW, Boyle DL, Tak PP, Gerlag DM, Wang W et al. DNA methylome signature in early rheumatoid arthritis synoviocytes compared with longstanding rheumatoid arthritis synoviocytes. Arthritis Rheum. 2015 doi:10.1002/art.39123

\section{Submit your next manuscript to BioMed Central and take full advantage of:}

- Convenient online submission

- Thorough peer review

- No space constraints or color figure charges

- Immediate publication on acceptance

- Inclusion in PubMed, CAS, Scopus and Google Scholar

- Research which is freely available for redistribution 